\title{
Loss of CclA, required for histone 3 lysine 4 methylation, decreases growth but increases secondary metabolite production in Aspergillus fumigatus
}

Secondary metabolite (SM) production in filamentous fungi is mechanistically associated with chromatin remodeling of specific SM clusters. One locus recently shown to be involved in SM suppression in Aspergillus nidulans was CclA, a member of the histone 3 lysine 4 methylating COMPASS complex. Here we examine loss of CclA and a putative H3K4 demethylase, HdmA, in the human pathogen Aspergillus fumigatus. Although deletion of $h d m A$ showed no phenotype under the conditions tested, the cclA deletant was deficient in tri- and di-methylation of H3K4 and yielded a slowly growing strain that was rich in the production of several SMs, including gliotoxin. Similar to deletion of other chromatin modifying enzymes, $\Delta c c l A$ was sensitive to 6-azauracil indicating a defect in transcriptional elongation. Despite the poor growth, the $\Delta c c l A$ mutant had wild-type pathogenicity in a murine model and the Toll-deficient Drosophila model of invasive aspergillosis. These data indicate that tri- and di-methylation of H3K4 is involved in the regulation of several secondary metabolites in A. fumigatus, however does not contribute to pathogenicity under the conditions tested. 
1 Jonathan M. Palmer ${ }^{1 *}$, Jin Woo Bok ${ }^{1 *}$, Seul Lee ${ }^{1 a}$, Taylor R.T. Dagenais ${ }^{1 b}$, David R. 2 Andes $^{1}$, Dimitrios P. Kontoyiannis ${ }^{3}$, and Nancy P. Keller ${ }^{1,2} \#$.

3

41 Department of Medical Microbiology and Immunology, University of 5 Wisconsin-Madison, Madison, WI, USA

$6{ }^{2}$ Department of Bacteriology, University of Wisconsin-Madison, Madison, WI, USA

$7{ }^{3}$ Department of Infectious Disease, University of Texas MD Anderson Cancer Center, 8 Houston, TX, USA

9

10 * Authors contributed equally to this work.

11 a current address: 103-1503 330 Homiro, Sangdang-Gu, Cheongju, Chungbuk, Korea

$12{ }^{\mathrm{b}}$ current address: Madison Area Technical College, 3550 Anderson St, Madison, 53704

13

14 \# corresponding author:

15 Nancy Keller

163476 Microbial Sciences Building

171550 Linden Drive

18 Madison, WI 53706

19 npkeller@wisc.edu

20 Phone: 1 (608) 262-9795

21 Fax: 1 (608) 262-8418

22 


\section{Introduction}

2 Aspergillus fumigatus is a saprophytic filamentous fungus commonly found in diverse

3 environments throughout the world. Whereas this fungus appears to have always been

4 associated with aspergillomas since such structures were recognized by medical

5 science in the 1800 s, the incidence of invasive aspergillosis (IA) is a more recent

6 phenomenon directly correlated to the increase in immune-compromised patients

7 originally treated for other diseases. Why $A$. fumigatus predominates as the causative

8 agent of IA instead of the 200 or so other Aspergilli is a question of considerable interest

9 as the answers should help in devising strategies to control this often fatal disease.

11 A. fumigatus possesses several attributes that are thought to contribute to its

12 predominance in IA and other Aspergillus-associated diseases; this topic has been the

13 subject of several recent reviews (Dagenais \& Keller 2009; Abad et al. 2010;

14 McCormick, Loeffler \& Ebel 2010). The reviews indicate that this species may not

15 possess unique classical virulence factors, but rather orchestrates the expression of

16 conserved fungal characteristics in such a way as to evade or overcome host defenses

17 more so than other Aspergilli. There are, however, at least two attributes that have been

18 associated with $A$. fumigatus virulence that vary considerably among Aspergillus

19 species: its remarkable thermo-tolerance and its unique repertoire of toxic secondary

20 metabolites (SM) (Dagenais \& Keller 2009; Abad et al. 2010).

22 Many studies dating back almost 30 years have implicated several toxins as important in 23 host - Aspergillus interactions (Mullbacher, Waring \& Eichner 1985; Eichner et al. 1986;

24 Sutton et al. 1994; Amitani et al. 1995; Khoufache et al. 2007; Fallon, Reeves \&

25 Kavanagh 2011). In recent years, individual genes required for SM production have

26 been removed from the $A$. fumigatus genome and the resultant deletants tested for 
1 virulence in various animal models (D'Enfert et al. 1996; Reeves et al. 2006; Ben-Ami et

2 al. 2009). Through these studies it was discovered that a global regulator of SM

3 production, LaeA, was shown to be important in IA development (Bok et al. 2005; Sugui

4 et al. 2007a). Furthermore, the LaeA regulated SM gliotoxin contributes to A. fumigatus

5 virulence in several animal models of IA (Reeves et al. 2004; Stanzani et al. 2005;

6 Cramer et al. 2006; Coméra et al. 2007; Sugui et al. 2007b; Ben-Ami et al. 2009;

7 Lionakis \& Kontoyiannis 2010). Since then, global regulation of SM gene clusters has

8 been found to be regulated in part through global changes in histone modifications,

9 particularly acetylation and methylation of histone 3 tail residues (Bok et al. 2005) and

10 reviewed in (Strauss \& Reyes-Domínguez 2011). These modifications have been linked

11 to LaeA activity (Reyes-Dominguez et al. 2010). A recent report in A. nidulans showed

12 that CcIA, a member of the conserved eukaryotic COMPASS complex that methylates

13 histone 3 lysine 4 (H3K4), was also critical in the regulation of several SMs (Bok et al.

14 2009). H3K4 can have up to five different modifications: acetylated, unmodified,

15 mono-methylated, di-methylated, and tri-methylated. Histone tail modifications were

16 once thought to be permanent, however more recent data has indicated that these

17 modifications are dynamic, i.e. they are reversible. For example, while the COMPASS

18 complex methylates H3K4 there is a reciprocal enzyme that demethylates H3K4. In

19 mammalian systems, a flavin containing amine oxidase named LSD1 (lysine specific

20 demethylase 1) is responsible for demethylation of H3K4 (Shi et al. 2004).

22 Based on our previous research in the model fungus $A$. nidulans, we identified the $A$.

23 fumigatus cClA and LSD1 homologs from the genome sequence. To investigate a 24 possible role of H3K4 methylation in SM cluster regulation and pathogenicity in $A$.

25 fumigatus, we deleted both $c c / A$ and the putative LSD1 homolog, which we named $26 h d m A$ (histone demethylase A). Whereas the $h d m A$ deletant was indistinguishable from 
1 wild type, the cclA deletant, similar to the phenotype of the $A$. nidulans $\triangle c c l A$ mutant,

2 was deficient in tri- and di-methylation of $\mathrm{H} 3 \mathrm{~K} 4$ and exhibited slow growth but increased

3 activation and expression of several $A$. fumigatus SMs, including gliotoxin. The mutant

4 showed wild type pathogenicity in both a neutropenic murine model and Toll-deficient

5 Drosophila model of invasive aspergillosis. These data suggest that tri- and

6 di-methylation of H3K4 is not required for pathogenicity under the conditions tested and

7 raises the possibility that pathogenicity of a slow growing mutant strain may be

8 compensated by enhanced gliotoxin synthesis.

\section{Materials and Methods.}

\section{Strains, growth conditions, and DNA manipulations}

13 All $A$. fumigatus strains used in this study are derivatives of the clinical isolate AF293

14 (Xue et al. 2004) and are listed in Table 1. Strains were maintained on glucose minimal 15 media (GMM) (Shimizu \& Keller 2001) and when appropriate the media was 16 supplemented with $5 \mathrm{mM}$ uracil and $5 \mathrm{mM}$ uridine for pyrG1 strains, $5 \mathrm{mM}$ arginine for $17 \operatorname{argB1}$ strains, and hygromycin was used at a final concentration of $100 \mathrm{ug} / \mathrm{ml}$. Radial 18 growth experiments and 6-azauracil sensitivity assays were carried out essentially as in 19 (Palmer et al. 2008). All DNA manipulations were conducted according to standard 20 protocols (Sambrook \& Russell 2001) and fungal transformation was done as previously 21 described (Miller, Miller \& Timberlake 1985) with a minor modification of protoplasts 22 being plated in $0.75 \%$ molten top agar. Primers used in this study are listed in Table 2.

\section{Construction of mutant $A$. fumigatus strains}

25 An $A$. fumigatus cclA disruption cassette was constructed using double-joint PCR 26 consisting of the following: 1-kb DNA fragment upstream of the $c c / A$ start codon (primers 
1 Fbre5F and Fbre5R-EcoRI), a 3-kb EcoRI-HindIII DNA fragment of $A$. parasisitus pyrG 2 via pJW24 (Calvo et al. 2004), and a 1-kb DNA fragment downstream of the cclA stop 3 codon (primers Fbre3F and Fbre3R-HindIII). The fusion PCR product was then used to 4 transform AF293.1 to prototrophy creating TJW84.1. Homologous single-gene 5 replacement of $c c / A$ was confirmed by Southern analysis (Fig. $1 \mathrm{~A}$ and B). In order to 6 complement the $\triangle c c / A$ mutant, a complementation plasmid (pJW109.5) was constructed.

7 The plasmid contained a 3.8-kb wild-type $c c / A$ gene including a 1-kb native promoter

8 and a 1-kb native termination cassette, which was amplified by primers FbreCOMF-Notl

9 and FbreCOMR-Spel. The PCR product wascloned into a Notl-Spel site of pUCH2-8

10 (Alexander. Hohn \& McCormick 1998), which contains the selectable marker hygromycin

11 B phosphotransferase. TJW84.1 was transformed with pJW109.5 to hygromycin

12 resistance and Southern blot confirmed single copy integration for TJW88.21 (Fig. 1B).

14 An hdmA disruption cassette was constructed by PCR amplifying a 1.1-kb DNA fragment upstream of the hdmA ORF using the primer pair JP1 and JP2 containing EcoRI restriction sites. The subsequent 1.1-kb EcoRI-EcoRI fragment was cloned into pJW24

17 to create pJMP1. A downstream 1.0-kb fragment was amplified using the primer pair 18 JP3 and JP4 containing Xbal and Notl restriction sites respectively. The 1.0-kb 19 Xbal-Notl fragment was cloned into pJMP1 to create the hdmA disruption cassette 20 named pJMP2. The hdmA ORF was disrupted by transformation of linearized pJMP2 21 into AF293.6 creating the $\triangle h d m A$ argB1 auxotrophic strain (TJMP2.36), which was 22 confirmed by Southern blot (Fig. 1C and D). In order to generate a prototrophic deletion 23 strain, an $\arg B$ complementation plasmid was constructed by amplifying a 2.6-kb 24 fragment corresponding to A. fumigatus argB using the primer pair 'JP Afumi argB For' 25 and 'JP Afumi argB Rev', which was subsequently cloned into pCR2.1 TOPO 26 (Invitrogen) to create pJMP4. This plasmid was transformed into TJMP2.36 to generate 
1 a prototrophic $\Delta h d m A$ strain (TJMP3.52). For complementation of $\Delta h d m A$, a $4.6-k b$

2 fragment corresponding to $\sim 0.9-\mathrm{kb}$ up stream and $\sim 0.5-\mathrm{kb}$ downstream of the $h d m A$

3 ORF was amplified with the primer pair 'JP IsdA comp For' and 'JP IsdA comp Rev' each

4 containing a BamHI site. The subsequent BamHI-BamHI fragment was cloned into

5 pJMP4 to create pJMP13. TJMP2.36 was transformed with pJMP13 to create a

6 complemented control strain TJMP29.1 $(\Delta h d m A+h d m A)$ and single integration was

7 confirmed by Southern blot (Fig. 1E).

8

9 Physiology experiments

10 Radial growth measurements and sensitivity to 6-azauracil (6AU) was conducted as

11 described previously (Palmer et al. 2008). Four replicates were used for each assay and

12 statistical significance was calculated with ANOVA analysis using Prism 5 software

13 (Graph Pad).

14

\section{Alterations in H3K4 methylation}

16 Nuclear extracts were isolated as previously described (Palmer et al. 2008).

17 Approximately $50 \mu \mathrm{g}$ of nuclear protein extract was electrophoresed on a $10 \%$

18 Tricine-SDS-PAGE gel (Schägger 2006) and subsequently electroblotted to

19 nitrocellulose membranes. Detection of H3K4 modifications was conducted using the

20 following primary antibodies and dilutions: 1:1,000 anti- histone 3 (Upstate, \#07-690),

21 1:1,000 anti- H3K4 mono-methylation (Upstate, \#07-436), 1:2,000 anti- H3K4

22 di-methylation (Upstate, \#07-030), 1:2,000 anti- H3K4 tri-methylation (Upstate, \#07-473).

23 Chemiluminescent detection was employed using SuperSignal West Pico

24 Chemiluminescent Substrate (Thermo Scientific) and a secondary goat 25 anti-rabbit-horseradish peroxidase conjugate antibody (Pierce, \#31460) diluted 26 1:15,0000 (Fig. 2). 


\section{Northern analysis and secondary metabolite extraction}

3 Fifty milliliter cultures of liquid GMM $+0.1 \%$ yeast extract were inoculated with $1 \times 10^{7}$

4 spores per $\mathrm{ml}$ and incubated at $250 \mathrm{rpm} 29^{\circ} \mathrm{C}$ for 24 hours, followed by a reduction in

5 temperature to $25^{\circ} \mathrm{C}$ for an additional 48 hours. Mycelia were harvested, lyophilized

6 overnight, and total RNA was extracted using Isol-RNA Lysis Reagent (5 Prime)

7 according to manufacturers recommendations. Subsequent northern analysis was done

8 using radiolabeled probes for the corresponding transcript (Fig. 4C) (primers are listed in

9 Table 2). Secondary metabolites were extracted from 20 milliliters of culture filtrate with

10 an equal volume of chloroform. The air-dried chloroform layer was resuspended in $75 \mu \mathrm{l}$

11 chloroform and $5 \mu \mathrm{l}$ was separated on a thin layer chromatography plate (Whatman,

12 \#4410 222) using chloroform: acetone (7:3) as a solvent. The TLC plate was then dried

13 and imaged under $254 \mathrm{~nm}$ and $366 \mathrm{~nm}$ ultraviolet light (Fig. 4A). Organic extracts from

14 solid minimal medium cultures was accomplished by taking equal sized cores from the

15 center of point-inoculated cultures, homogenized in 3 milliliters of water, and extracted

16 with an equal volume of chloroform. Quantification of TLC spots was achieved through

17 densitometry analysis using Image $\mathrm{J}$ and normalized to wild type levels.

19 Pathogenicity Assays

20 The neutropenic murine model of aspergillosis was conducted as described previously

21 (Bok et al. 2005) with approval of the William S. Middleton VA and the University of

22 Wisconsin Animal Care Committees in accordance with the MV2344 animal use

23 protocol. Toll-deficient flies were generated by crossing flies carrying a thermosensitive

24 allele of Toll (TI r632) with flies carrying a null allele of Toll (TI I-RXA) (Lionakis et al.

25 2005). Two- to four day old adult female Toll-deficient flies were used in all of the

26 experiments. Twenty flies were infected with each $A$. fumigatus strain used in this study. 
1 All of the experiments were performed in triplicate. To minimize circadian rhythm

2 variability, all experiments were performed at 9 AM. A. fumigatus isolates were grown on 3 yeast extract agar glucose (YAG) at $37^{\circ} \mathrm{C}$. Conidia were collected in sterile $0.9 \%$ saline 4 from 2 days old cultures. The conidial concentration suspension was determined by 5 using a hemacytometer and adjusted to $1 \times 10^{7}$ per $\mathrm{ml}$. The dorsal side of the thorax of 6 twenty $\mathrm{CO}_{2}$ anesthetized flies was punctured with a thin $(10 \mu \mathrm{m})$ sterile needle that had 7 been dipped in a concentrated solution of $A$. fumigatus conidia $\left(10^{7}\right.$ cells $\left./ \mathrm{ml}\right)$. In our 8 previous work, this method was shown to deliver a reproducible inoculum of 700 to 800 9 conidia (Lionakis et al. 2005). As a negative control group, Toll-deficient flies were 10 punctured with a $10 \mu \mathrm{m}$ sterile needle and monitored daily for survival. Flies that died 11 within $3 \mathrm{~h}$ of the injection were considered to have died as a result of the puncture 12 procedure and were not included in the survival rate analysis. The flies were housed in 13 a $29^{\circ} \mathrm{C}$ incubator to maximize expression of the $\mathrm{Tl}$ r632 phenotype (Lemaitre et al. 14 1996). The Toll-deficient flies were transferred into fresh vials every 3 days. Fly survival 15 was assessed daily over 8 days.

16

17

Results.

Deletion of cclA but not hdmA affects global histone 3 lysine 4 methylation patterns.

Aspergillus fumigatus cclA was identified through BLASTp analysis of the A. fumigatus genome with the A. nidulans CclA amino acid sequence (Bok et al. 2009), which yielded one homolog Afu3g04120. The human LSD1 protein sequence (Genbank, \#060341) was used to BLASTp the $A$. fumigatus genome sequence, which provided one potential ortholog, Afu4g13000. Afu4g13000 is $29 \%$ identical to human LSD1 and contains the 
1 conserved SWIRM domain, a flavin amine oxidase domain, as well as an HMG Box

2 domain (Shi et al. 2004) and thus was named hdmA for histone demethylase $\underline{\text { A. }}$.

4 The cclA and hdmA alleles were independently disrupted in the A. fumigatus AF293

5 genetic background. Southern analysis of transformants for each gene confirmed simple

6 gene replacements (Fig. 1). One representative deletant, TJW84.1 for $\Delta c c / A$ and

7 TJMP3.52 for $\Delta h d m A$, was chosen for each gene replacement for the studies presented

8 below. Both deletants were complemented with their respective genes to generate a

9 single copy $c c l A+\Delta c c l A$ strain and a single copy $h d m A+\Delta h d m A$ strain as shown in

10 Table 1.

12 Our first experiment was to assess if loss of either gene affected histone 3 lysine 4 13 methylation (H3K4). The $\triangle$ cclA strain showed a clear loss of whole genome H3K4 14 tri-methylation and a considerable decrease in di-methylation (Fig. 2); this tallied with 15 earlier studies in our lab assessing $\mathrm{H} 3 \mathrm{~K} 4$ methylation in an $A$. nidulans $\Delta c c / A$ mutant 16 (Bok et al. 2009). These data are also consistent with BRE2 mutants in Saccharomyces

17 cerevisiae, where loss of BRE2 results in strains with diminished di-methylation and no 18 tri-methylation of H3K4 (Schneider et al. 2005). The complemented strain was restored 19 in H3K4 methylation to wild type levels. However, loss of hdmA did not exhibit a 20 detectable impact on whole genome H3K4 methylation (Fig. 2).

\section{CcIA mutants are crippled in growth and sensitive to 6-Azauracil.}

23 As reported for the $A$. nidulans $\Delta c c / A$ mutant (Giles et al. 2011), the $A$. fumigatus $\Delta c c / A$

24 strain exhibited poor growth as exhibited by decreased radial growth on several different 25 media, including rich media (YPD and Champs) as well as our standard minimal medium 26 (GMM) (Fig. 3A). Additionally, the $\triangle c c l A$ mutant had a measurable growth defect at all 
1 temperatures tested (Fig. 3B) and decreased mass when grown in liquid shaking culture

2 (data not shown). This defect was rescued in the complemented strains. Neither the

$3 h d m A$ deletant nor its complement exhibited any growth phenotype.

5 The phenotype of the $\Delta c c l A$ strain shared some similarities to that of a previously

6 described $A$. fumigatus chromatin mutant, $\Delta c / r D$ (Palmer et al. 2008), impaired in

7 methylation of H3K9. As histone methyltransferase mutants are often found to be

8 sensitive to chemicals that target various cellular processes, the $\Delta c / r D$ mutant had been

9 assessed for sensitivity to various chemical agents and found to be sensitive to

10 6-azauracil (6AU); an inhibitor of guanine nucleotide synthesis and indicator of

11 transcriptional defects (Riles et al. 2004; Zhang et al. 2005; Palmer et al. 2008).

12 Following that protocol, the $\Delta c c / A$ and $\Delta h d m A$ strains were similarly screened and the

13 former but not the latter found to be also sensitive to 6AU (Fig. 3C). Resistance to 6AU

14 was rescued in the complement strain. Moreover, we did not find differences in

15 sensitivity of $\Delta h d m A$ or $\Delta c c l A$ strains compared to wild type when exposed to several

16 chemical agents that included hydroxyurea (DNA synthesis inhibitor), thiabendazole

17 (microtubule destabilizer), $1.2 \mathrm{M}$ Sorbitol (osmotic stress), $0.6 \mathrm{M} \mathrm{KCl}$ (osmotic stress),

18 Congo red (cell wall inhibitor), or Calcofluor white (cell wall inhibitor) (data not shown).

20 CclA loss results in increased secondary metabolism.

21 Mutations in chromatin remodeling genes have frequently been associated with

22 alterations in secondary metabolite production, both in A. nidulans (Shwab et al. 2007;

23 Bok et al. 2009) and $A$. fumigatus (Lee et al. 2009). In particular the $A$. nidulans $\Delta c c / A$

24 strain was found to produce novel metabolites with antimicrobial properties (Bok et al.

25 2009; Giles et al. 2011). An examination of extracts from three different conditions of the

26 two mutants showed that the $A$. fumigatus $\Delta c c l A$ strain but not the $\Delta h d m A$ strain was 10 
1 increased in the production of several metabolites (Fig. 4). In particular, this strain

2 produced over 4 times as much gliotoxin compared to wild type when grown in liquid

3 shaking culture (Fig. 4A and B). The high level of gliotoxin was associated with a large

4 increase in transcription of gliz, the $\mathrm{C} 6$ transcription factor required for gli expression

5 (Fig. 4C). Under these growth conditions, loss of $c c / A$ also resulted in several fold

6 increases of numerous other metabolites (Fig. $4 \mathrm{~A}$ and B). The $\Delta c c / A$ mutant was also

7 analyzed under alternative growth conditions and produces similar amounts of

8 metabolites on solid medium at $29^{\circ} \mathrm{C}$ compared to wild type (Fig. 4D), however $\Delta c c / A$

9 mutants produce more of a few unidentified metabolites when grown $37^{\circ} \mathrm{C}$ on solid

10 medium (Fig. 4D).

12 Virulence of $\Delta c c l A$ is unaltered in a mammalian and Drosophila model.

13 Although poorly growing $A$. fumigatus mutants have often been found to exhibit a 14 decrease in virulence (D'Enfert et al. 1996; Brown et al. 2000), the increased toxin 15 production in the $\Delta c c / A$ strain coupled with the poor growth presented a unique 16 phenotype that disallowed an obvious prediction on its pathogenicity attributes.

17 Therefore, to assess $c c l A$ or hdmA loss on virulence, we examined virulence in two 18 animal models. Assessment of the mutants in a neutropenic murine model showed no 19 significant difference between the wild type and mutant strains (Fig. 5A). This was also 20 true for the Toll-deficient Drosophila model (where only $\triangle c c / A$ was assessed, Fig. 5B).

\section{Discussion.}

25 Genes involved in secondary metabolism in fungi are arranged in a clustered format that 26 is easily impacted by chromatin level alterations (Palmer \& Keller 2010). This 
1 characteristic has been illustrated primarily by two ways: either through manipulation

2 (deletion or overexpression) of chromatin modifying genes or SM induction via chemical

3 epigenetics (Bok et al. 2009; Cichewicz 2010). LaeA, a global regulator of SM

4 (Reyes-Dominguez et al. 2010), is a conserved virulence factor in filamentous fungi

5 including A. fumigatus, A. flavus and Gibberella zeae (Bok et al. 2005; Sugui et al.

6 2007a; Amaike \& Keller 2009). Although the mechanism of LaeA action is not known,

7 the results of its activity, such as decrease in heterochromatin marks (e.g. H3K9

8 tri-methylation, (Reyes-Dominguez et al. 2010)) correlated with increased SM cluster

9 expression, are thought to contribute to its role as a virulence factor in IA. Extrapolation

10 of LaeA impact on chromatin led to the hypothesis that other chromatin remodeling

11 genes, such as histone methyltransferases, acetylases and their cognate demethylases

12 and deacetylases would also impact SM production and, possibly, virulence attributes in

13 pathogenic fungi such as $A$. fumigatus.

14

15 Here our efforts focused on two conserved genes that in other systems are involved in 16 methylation and demethylation of histone 3 on lysine 4. The methylation state of H3K4

17 is associated with both gene activation and repression in eukaryotes (Krogan et al. 2002;

18 Mueller, Canze \& Bryk 2006). The conserved eukaryotic COMPASS complex is required

19 for methylation of H3K4 with one of its key members a SPRY domain protein termed

20 Bre2p in Saccharomyces cerevisiae (Krogan et al. 2002), Ash2p in

21 Schizosaccharomyces pombe (Roguev et al. 2001) and CclA in A. nidulans (Bok et al.

22 2009). Demethylation of H3K4 is achieved through activity of an amine oxidase called

23 LSD1 in higher eukaryotes (Shi et al. 2004; Shi et al. 2005). Aspergillus species contain

24 one putative LSD1 homolog, which we termed $h d m A$ in this work due to the existence of

25 a previously named IsdA gene - containing no similarity to $h d m A$ - involved in late sexual

26 development in A. nidulans (Lee et al. 2001). 
2 Our previous results in $A$. nidulans demonstrated a critical role for CcIA in both SM

3 production as well as normal growth (Bok et al. 2009; Giles et al. 2011). This phenotype

4 was replicated in the $A$. fumigatus $\Delta c c l A$ mutant. On the other hand, the $h d m A$ deletant

5 in A. nidulans had a more subtle effect, and similar to what we describe here for the $A$.

6 fumigatus $\Delta$ dmA mutant, small impact on fungal morphology (data not shown).

7 Notably, deletion of $c c / A$ in either species was accompanied by an easily detectable

8 decrease in H3K4 methylation, however under the conditions used in this study, no

9 difference in H3K4 methylation was observed in the $\triangle h d m A$ strain. Deletion or down

10 regulation of this protein in other organisms can be detected by increased H3K4

11 methylation (Shi et al. 2004); possibly this increased H3K4 methylation is not observable

12 in bulk histone assessment as measured in this study or, alternatively, $\mathrm{HdmA}$ is not the

13 (major) H3K4 demethylase in $A$. fumigatus. An additional class of Jumonji C domain

14 (JmjC) containing proteins has been shown to demethylate lysine residues in histone

15 tails. While chromatin structure in the aspergilli is thought to be more similar to fission

16 yeast and higher eukaryotes, in S. cerevisiae a JmjC domain containing protein, Jhd2p,

17 is the major demethylase of H3K4 (Liang et al. 2007; Seward et al. 2007). Moreover

18 there is a Jhd2 homolog (Afu5g03430) present in the A. fumigatus genome and thus

19 perhaps Afu5g03430 is the major demethylase of H3K4 in the aspergilli.

21 Stress assays suggested that the $\triangle c c l A$ strain was more sensitive to $6 \mathrm{AU}$, a chemical 22 used to identify strains impaired in transcriptional processes, than wild type or the 23 complemented strain. Use of this chemical has identified several S. cerevisiae histone 24 methyltransferase mutants defective in transcriptional elongation (Exinger \& Lacroute 25 1992; Li, Moazed \& Gygi 2002; Zhang et al. 2005), therefore it is perhaps not surprising 26 that two $A$. fumigatus histone methyltransferase mutants - $\Delta c c l A$ described in this study 13 
1 and $\Delta c / r D$ previously described - are also sensitive to this chemical. Whereas the

2 mechanism of $\triangle c c l A$ sensitivity to $6 A U$ is unknown, this sensitivity could reflect a defect

3 in the fungal transcriptional machinery in this strain, which highlights the pleiotropic

4 phenotypes of these mutants.

5

6 The increased SM output from the $\triangle c c / A$ strain (Fig. 4) likely arises from aberrancies in

7 transcriptional activity, in this case primarily enhancing transcription of a subset of SM

8 clusters as previously demonstrated in the A. nidulans $\Delta$ cclA mutant (Bok et al. 2009).

9 The increased expression of gliz correlated well to the significant increase in gliotoxin

10 synthesis in this strain. Whereas we have not characterized the other up regulated

11 metabolites in this strain, we speculate that some of them may be a result of 'turning on'

12 formerly silent SM clusters and we will be investigating this hypothesis in future studies.

14 In A. fumigatus, mutants that have been shown to display poor growth in vitro have also

15 been shown to be less virulent in model of IA (D'Enfert et al. 1996; Brown et al. 2000).

16 However, it was recently shown in Candida albicans that mutants that grow poorly in

17 vitro do not always show a decrease in virulence (Noble et al. 2010). Similarly, 18 disruption of ClrD in $A$. fumigatus results in a mutant that is defective in growth and 19 asexual sporulation (Palmer et al. 2008) however did not result in a decrease in 20 pathogenicity in the mouse model of IA (T. Dagenais, D. Andes \& N. Keller, unpublished 21 data). Thus, the pleiotropic phenotype of poor growth in the laboratory with increased 22 secondary metabolite synthesis of the $\Delta c c / A$ mutant presented an interesting and 23 potentially opposing coupling of putative $A$. fumigatus virulence attributes, as enhanced 24 gliotoxin production would be expected to increase virulence (Bok et al. 2006; Cramer et 25 al. 2006; Sugui et al. 2007b). The results from our studies suggest that loss of the CclA 26 gene had no significant effect on pathogenicity in either a neutropenic murine or 
1 Drosophila model of IA. Although we did not test growth rate in the host organisms, our

2 data could suggest that pathogenicity can be restored in poor growing strains by

3 increased secondary metabolite (gliotoxin) production, or alternatively, could indicate

4 that genetic factors regulating in vitro growth may differ from those controlling in vivo

5 growth. Moreover, this study supports the view that a composition of many A. fumigatus

6 characteristics contributes to the pathogenicity of this species.

7

8

9

10

11

12

13

14

15

16

17

18

19

20

21

22

23

24

25

26

27

28

29

30

31

32

33

34

\section{References}

Abad A, Victoria Fernández-Molina J, Bikandi J, Ramírez A, Margareto J, Sendino J, Luis Hernando F, Pontón J, Garaizar J, Rementeria A. 2010. What makes Aspergillus fumigatus a successful pathogen? Genes and molecules involved in invasive aspergillosis. Revista Iberoamericana de Micologia, 27, 155-182.

Alexander NJ, Hohn TM, Mccormick SP. 1998. The TR/11 gene of Fusarium sporotrichioides encodes a cytochrome P-450 monooxygenase required for C-15 hydroxylation in trichothecene biosynthesis. Applied and Environmental Microbiology, 64, 221-225.

Amaike S, Keller NP. 2009. Distinct roles for VeA and LaeA in development and pathogenesis of Aspergillus flavus. Eukaryotic Cell, 8, 1051-1060.

Amitani R, Taylor G, Elezis EN, Llewellyn-Jones C, Mitchell J, Kuze F, Cole PJ, Wilson R. 1995. Purification and characterization of factors produced by Aspergillus fumigatus which affect human ciliated respiratory epithelium. Infection and Immunity, 63, 3266-3271.

Ben-Ami R, Lewis RE, Leventakos K, Kontoyiannis DP. 2009. Aspergillus fumigatus inhibits angiogenesis through the production of gliotoxin and other secondary metabolites. Blood, 114, 5393-5399.

Bok JW, Balajee SA, Marr KA, Andes D, Nielsen KF, Frisvad JC, Keller NP. 2005. LaeA, a regulator of morphogenetic fungal virulence factors. Eukaryotic Cell, 4, 1574-1582. 
1 Bok JW, Chiang Y-M, Szewczyk E, Reyes-Dominguez Y, Davidson AD, Sanchez JF, Lo H-C, Watanabe K, Strauss J, Oakley BR, Wang CCC, Keller NP. 2009. Chromatin-level regulation of biosynthetic gene clusters. Nature Chemical Biology, 5, 462-464.

Bok JW, Chung D, Balajee SA, Marr KA, Andes D, Nielsen KF, Frisvad JC, Kirby KA, Keller NP. 2006. Gliz, a transcriptional regulator of gliotoxin biosynthesis, contributes to Aspergillus fumigatus virulence. Infection and Immunity, 74, 6761-6768.

Brown JS, Aufauvre-Brown A, Brown J, Jennings JM, Arst H, Holden DW. 2000. Signature-tagged and directed mutagenesis identify PABA synthetase as essential for Aspergillus fumigatus pathogenicity. Molecular Microbiology, 36, 1371-1380.

Calvo AM, Bok JW, Brooks W, Keller NP. 2004. veA is required for toxin and sclerotial production in Aspergillus parasiticus. Applied and Environmental Microbiology, 70, 4733-4739.

Cichewicz RH. 2010. Epigenome manipulation as a pathway to new natural product scaffolds and their congeners. Natural Product Reports, 27, 11-22.

Coméra C, André K, Laffitte J, Collet X, Galtier P, Maridonneau-Parini I. 2007. Gliotoxin from Aspergillus fumigatus affects phagocytosis and the organization of the actin cytoskeleton by distinct signaling pathways in human neutrophils. Microbes and Infection, 9, 47-54.

Cramer RA, Gamcsik MP, Brooking RM, Najvar LK, Kirkpatrick WR, Patterson TF, Balibar CJ, Graybill JR, Perfect JR, Abraham SN, Steinbach WJ. 2006. Disruption of a nonribosomal peptide synthetase in Aspergillus fumigatus eliminates gliotoxin production. Eukaryotic Cell, 5, 972-980.

D'enfert C, Diaquin M, Delit A, Wuscher N, Debeaupuis JP, Huerre M, Latgé JP. 1996. Attenuated virulence of uridine-uracil auxotrophs of Aspergillus fumigatus. Infection and Immunity, 64, 4401-4405.

Dagenais TRT, Keller NP. 2009. Pathogenesis of Aspergillus fumigatus in invasive aspergillosis. Clinical Microbiology Reviews, 22, 447-465.

Eichner RD, Al Salami M, Wood PR, Mullbacher A. 1986. The effect of gliotoxin upon macrophage function. International Journal of Immunopharmacology, 8, 789-797.

Exinger F, Lacroute F. 1992. 6-Azauracil inhibition of GTP biosynthesis in Saccharomyces cerevisiae. Current Genetics, 22, 9-11.

Fallon JP, Reeves EP, Kavanagh K. 2011. The Aspergillus fumigatus toxin fumagillin suppresses the immune response of Galleria mellonella larvae by inhibiting the action of haemocytes. Microbiology, 157, 1481-1488. 
1 Giles SS, Soukup AA, Lauer C, Shaaban M, Lin A, Oakley BR, Wang CCC, Keller NP. 2011. Cryptic Aspergillus nidulans antimicrobials. Applied and Environmental Microbiology, 77, 3669-3675.

Khoufache K, Puel O, Loiseau N, Delaforge M, Rivollet D, Coste A, Cordonnier C, Escudier E, Botterel F, Bretagne S. 2007. Verruculogen associated with Aspergillus fumigatus hyphae and conidia modifies the electrophysiological properties of human nasal epithelial cells. BMC Microbiology, 7, 5 .

Krogan NJ, Dover J, Khorrami S, Greenblatt JF, Schneider J, Johnston M, Shilatifard A. 2002. COMPASS, a histone H3 (Lysine 4) methyltransferase required for telomeric silencing of gene expression. Journal of Biological Chemistry, 277, 10753-10755.

Lee DW, Kim S, Kim SJ, Han DM, Jahng KY, Chae K-S. 2001. The IsdA gene is necessary for sexual development inhibition by a salt in Aspergillus nidulans. Current Genetics, 39, 237-243.

Lee I, Oh J, Shwab EK, Dagenais T, Andes D, Keller N. 2009. HdaA, a class 2 histone deacetylase of Aspergillus fumigatus, affects germination and secondary metabolite production. Fungal Genetics and Biology, 46, 782-790.

Lemaitre B, Nicolas E, Michaut L, Reichhart JM, Hoffmann JA. 1996. The dorsoventral regulatory gene cassette spätzle/Toll/cactus controls the potent antifungal response in Drosophila adults. Cell, 86, 973-983.

Li J, Moazed D, Gygi SP. 2002. Association of the histone methyltransferase Set2 with RNA polymerase II plays a role in transcription elongation. Journal of Biological Chemistry, 277, 49383-49388.

Liang G, Klose RJ, Gardner KE, Zhang Y. 2007. Yeast Jhd2p is a histone H3 Lys4 trimethyl demethylase. Nature Structural and Molecular Biology, 14, 243-245.

Lionakis MS, Kontoyiannis DP. 2010. The growing promise of Toll-deficient Drosophila melanogaster as a model for studying Aspergillus pathogenesis and treatment. Virulence, 1, 488-499.

Lionakis MS, Lewis RE, May GS, Wiederhold NP, Albert ND, Halder G, Kontoyiannis DP. 2005. Toll-deficient Drosophila flies as a fast, high-throughput model for the study of antifungal drug efficacy against invasive aspergillosis and Aspergillus virulence. Journal of Infectious Diseases, 191, 1188-1195.

Mccormick A, Loeffler J, Ebel F. 2010. Aspergillus fumigatus: contours of an opportunistic human pathogen. Cellular Microbiology, 12, 1535-1543.

Miller BL, Miller KY, Timberlake WE. 1985. Direct and indirect gene replacements in Aspergillus nidulans. Molecular and Cellular Biology, 5, 1714-1721.

Mueller JE, Canze M, Bryk M. 2006. The requirements for COMPASS and Paf1 in transcriptional silencing and methylation of histone $\mathrm{H} 3$ in Saccharomyces cerevisiae. Genetics, 173, 557-567. 
Mullbacher A, Waring P, Eichner RD. 1985. Identification of an agent in cultures of Aspergillus fumigatus displaying anti-phagocytic and immunomodulating activity in vitro. Journal of General Microbiology, 131, 1251-1258.

Noble SM, French S, Kohn LA, Chen V, Johnson AD. 2010. Systematic screens of a Candida albicans homozygous deletion library decouple morphogenetic switching and pathogenicity. Nature Genetics, 42, 590-598.

Palmer JM, Keller NP. 2010. Secondary metabolism in fungi: does chromosomal location matter? Current Opinion in Microbiology, 13, 431-436.

Palmer JM, Perrin RM, Dagenais TR, Keller NP. 2008. H3K9 methylation regulates growth and development in Aspergillus fumigatus. Eukaryotic Cell, 7, 2052-2060.

Reeves EP, Messina CGM, Doyle S, Kavanagh K. 2004. Correlation between gliotoxin production and virulence of Aspergillus fumigatus in Galleria mellonella. Mycopathologia, 158, 73-79.

Reeves EP, Reiber K, Neville C, Scheibner O, Kavanagh K, Doyle S. 2006. A nonribosomal peptide synthetase (Pes1) confers protection against oxidative stress in Aspergillus fumigatus. FEBS Journal, 273, 3038-3053.

Reyes-Dominguez Y, Bok JW, Berger H, Shwab EK, Basheer A, Gallmetzer A, Scazzocchio C, Keller NP, Strauss J. 2010. Heterochromatic marks are associated with the repression of secondary metabolism clusters in Aspergillus nidulans. Molecular Microbiology, 76, 1376-1386.

Riles L, Shaw RJ, Johnston M, Reines D. 2004. Large-scale screening of yeast mutants for sensitivity to the IMP dehydrogenase inhibitor 6-azauracil. Yeast, 21, 241-248.

Roguev A, Schaft D, Shevchenko A, Pijnappel WW, Wilm M, Aasland R, Stewart AF. 2001. The Saccharomyces cerevisiae Set1 complex includes an Ash2 homologue and methylates histone 3 lysine 4. EMBO Journal, 20, 7137-7148.

Sambrook J, Russell DW. 2001. Molecular Cloning: A Laboratory Manual. Cold Spring Harbor, NY: Cold Spring Harbor Laboratory Press.

Schägger H. 2006. Tricine-SDS-PAGE. Nature Protocols, 1, 16-22.

Schneider J, Wood A, Lee J-S, Schuster R, Dueker J, Maguire C, Swanson SK, Florens L, Washburn MP, Shilatifard A. 2005. Molecular regulation of histone H3 trimethylation by COMPASS and the regulation of gene expression. Molecular Cell, 19, 849-856.

Seward DJ, Cubberley G, Kim S, Schonewald M, Zhang L, Tripet B, Bentley DL. 2007. Demethylation of trimethylated histone H3 Lys4 in vivo by JARID1 JmjC proteins. Nature Structural and Molecular Biology, 14, 240-242.

Shi Y, Lan F, Matson C, Mulligan P, Whetstine JR, Cole PA, Casero RA, Shi Y. 2004. Histone demethylation mediated by the nuclear amine oxidase homolog LSD1. Cell, 119, 941-953. 
Shi YJ, Matson C, Lan F, Iwase S, Baba T, Shi Y. 2005. Regulation of LSD1 histone demethylase activity by its associated factors. Molecular Cell, 19, 857-864.

Shimizu K, Keller NP. 2001. Genetic involvement of a cAMP-dependent protein kinase in a $G$ protein signaling pathway regulating morphological and chemical transitions in Aspergillus nidulans. Genetics, 157, 591-600.

Shwab EK, Bok JW, Tribus M, Galehr J, Graessle S, Keller NP. 2007. Histone deacetylase activity regulates chemical diversity in Aspergillus. Eukaryotic Cell, 6, 1656-1664.

Stanzani M, Orciuolo E, Lewis RE, Kontoyiannis DP, Martins SL, St John LS, Komanduri KV. 2005. Aspergillus fumigatus suppresses the human cellular immune response via gliotoxin-mediated apoptosis of monocytes. Blood, 105, 2258-2265.

Strauss J, Reyes-Domínguez Y. 2011. Regulation of secondary metabolism by chromatin structure and epigenetic codes. Fungal Genetics and Biology, 48, 62-69.

Sugui JA, Pardo J, Chang YC, Müllbacher A, Zarember KA, Galvez EM, Brinster L, Zerfas P, Gallin JI, Simon MM, Kwon-Chung KJ. 2007a. Role of laeA in the regulation of alb1, gliP, conidial morphology, and virulence in Aspergillus fumigatus. Eukaryotic Cell, 6, 1552-1561.

Sugui JA, Pardo J, Chang YC, Zarember KA, Nardone G, Galvez EM, Müllbacher A, Gallin JI, Simon MM, Kwon-Chung KJ. 2007b. Gliotoxin is a virulence factor of Aspergillus fumigatus: gliP deletion attenuates virulence in mice immunosuppressed with hydrocortisone. Eukaryotic Cell, 6, 1562-1569.

Sutton P, Newcombe NR, Waring P, Mullbacher A. 1994. In vivo immunosuppressive activity of gliotoxin, a metabolite produced by human pathogenic fungi. Infection and Immunity, 62, 1192-1198.

Xue T, Nguyen CK, Romans A, Kontoyiannis DP, May GS. 2004. Isogenic auxotrophic mutant strains in the Aspergillus fumigatus genome reference strain AF293. Archives of Microbiology, 182, 346-353.

Zhang L, Schroeder S, Fong N, Bentley DL. 2005. Altered nucleosome occupancy and histone H3K4 methylation in response to 'transcriptional stress'. EMBO Journal, 24, 2379-2390. 


\section{Figures Legends}

3 Figure 1. Construction of deletion and subsequent complementation strains of

4 cclA and $h d m A$ in $A$. fumigatus. (A) A schematic drawn to scale illustrates the 5 replacement of the cclA ORF with the A. parasiticus pyrG gene and the location of Xhol 6 restriction enzyme sites. (B) A radiolabeled probe consisting of the cclA ORF and $\sim 1 \mathrm{~kb}$ 7 on either side was used to probe a Southern blot of Xhol digested genomic DNA. The 8 expected banding pattern was observed. Confirmation of a complemented cclA strain 9 (TJW88.21) was achieved via Southern blot using the described conditions above. An 10 additional band is shown that is greater than $10 \mathrm{~kb}$ that is attributed to the random 11 integration of the cclA complementing plasmid. (C) Schematic drawn to scale of the 12 restriction enzyme cut sites in the wild type and $\Delta h d m A$ backgrounds. (D) Southern blot 13 using a HindIII digestion and a Xbal-Pvul double digestion using a radiolabeled probe 14 consisting of the gene deletion construct shows the expected size differences between 15 wild type and the $\Delta h d m A$ strains. (E) Complementation of the $\Delta h d m A$ mutant was 16 confirmed using a Smal and subsequent HindIII digestion to determine single integration 17 of the complementing plasmid (TJMP29.1).

Figure 2. Deletion of cclA results in a reduction in di-methylation and loss of 20 tri-methylation of lysine 4 of histone 3 (H3K4). Western blot of nuclear protein 21 extracts clearly indicate that $c c / A$ is required for proper methylation of $\mathrm{H} 3 \mathrm{~K} 4$. While 22 H3K4 mono-methylation is unaffected by loss of cclA, di-methylation is reduced and 23 tri-methylation was absent. These data are consistent with S. cerevisiae BRE2 null 24 mutants. No difference in global methylation patterns of H3K4 was detected in the $25 \Delta h d m A$ strain compared to wild type. 
1 Figure 3. Null mutants of cclA result in reduced growth and increased sensitivity

2 to 6-azauracil. (A) The $\triangle c c l A$ strain is defective in radial growth on rich media (YPD,

3 Champs) as well as minimal media (GMM) using both nitrate and ammonium as a

4 nitrogen source. The complemented control strain restores wild-type levels of growth.

5 (B) Quantification of radial growth on solid media at three different temperatures $\left(25^{\circ} \mathrm{C}\right.$,

$629^{\circ} \mathrm{C}$, and $37^{\circ} \mathrm{C}$ ) illustrates a significant growth reduction in the $\Delta c c / A$ strain in all

7 conditions tested. No phenotype was observed for a mutant lacking hdmA. (C) Radial

8 growth assays conducted on GMM amended with $100 \mathrm{ug} / \mathrm{ml}$ of 6-azauracil indicated that

9 null mutants of $c c / A$ are more sensitive to 6-azauracil than wild type. The cclA

10 complemented control strain restores sensitivity to 6-azauracil to wild type levels.

11 Asterisk indicates $p<0.001$ using an ANOVA test for statistical significance with Prism 5

12 software.

13

14 Figure 4. CclA, but not HdmA, controls production of several secondary metabolites, including gliotoxin. (A) Strains were grown in liquid minimal media shaking culture and analyzed for production of secondary metabolites by thin layer

17 chromatography (TLC) using gliotoxin as a standard. UV infused TLC plates were 18 photographed under $254 \mathrm{~nm}$ and $366 \mathrm{~nm}$ light. (B) Quantification of TLC spots was 19 achieved by densitometry with Image $\mathrm{J}$ software and relative density was normalized to 20 wild type extracts, which reveals that null mutants of $c c / A$ produced more than 4 times 21 more gliotoxin as well as several fold more production of several unidentified 22 metabolites. (C) Northern analysis from the same growth conditions confirms the strains 23 as well as identifies a large increase in gliz transcript in the $\Delta c c / A$ mutant background 24 compared to wild type. There are no differences in transcription of the regulator of 25 secondary metabolism laeA. (D) Organic extracts were prepared from cultures grow on 26 solid media and subsequently analyzed via TLC analysis using chloroform:acetone (7:3) 
1 as a solvent. Growth at $29^{\circ} \mathrm{C}$ results in no detectable difference between $\Delta c c / A$ and wild

2 type, however at $37^{\circ} \mathrm{C}$ a few metabolites are slightly increased in $\Delta c c / A$ compared to wild 3 type.

4

5 Figure 5. Pathogenicity of $\Delta c c / A$ and $\Delta h d m A$ strains are wild type in murine model

6 of IA and $\triangle c c$ IA strains are wild type in Toll-deficient Drosophila model. (A)

7 Pathogenicity of WT, $\Delta c c l A, c c l A$ complement, $\Delta h d m A$, and $h d m A$ complement strains

8 was assessed using the neutropenic murine model of aspergillosis. No differences were

9 measured in any of the strains tested in comparison to wild type (AF293). (B) An

10 alternative pathogenicity assay using Toll-deficient fruit flies illustrates that the $\Delta c c / A$

11 mutant is no different than wild type. The Mantel-Cox statistical test was used to assess

12 differences in survival curves.

13 


\section{Table $\mathbf{1}_{\text {(on next page) }}$}

Strains used in this study. 
Table 1. Strains used in this study

\begin{tabular}{|c|c|c|}
\hline Strain & Genotype & Source \\
\hline AF293 & Wild type & (Xue et al. 2004) \\
\hline AF293.1 & pyrG1 & (Xue et al. 2004) \\
\hline AF293.6 & pyrG1, argB1 & (Xue et al. 2004) \\
\hline TJW84.1 & pyrG1, $\Delta c c l A::$ A. parasiticus pyrG & This study \\
\hline TJW88.21 & pyrG1, $\triangle c c \mid A::$ A. parsasiticus pyrG, cclA::hygromycin & This study \\
\hline TJMP2.36 & pyrG1, argB1, $\triangle h d m A: \because A$. parasiticus pyrG & This study \\
\hline TJMP3.52 & pyrG1, argB1, $\triangle h d m A:: A$. parasiiticus pyrG, A. fumigatus argB & This study \\
\hline TJMP29.1 & pyrG1, argB1, $\triangle h d m A:: A$. parasiticus pyrG, hdmA:A. fumigatus argB & This study \\
\hline
\end{tabular}




\section{Table 2 (on next page)}

Primers used in this study. 
Table 2. Primers used in this study

\begin{tabular}{|c|c|c|}
\hline Name & Sequence (5' to 3'): Restriction sites underlined & Purpose \\
\hline Fbre5F & ATATCAGTTGTTGCTCCTAGGGC & 5' Flank $\Delta c c l A$ \\
\hline Fbre5R-EcoRI & CATGTGGAATTCAATAACGGTTCACGAGTAAATTG & 5' Flank $\triangle c c l A$ \\
\hline Fbre3F-HindIII & TGGGGTAAGCTTGTTCCGAGCCATATCTGTC & 3' Flank $\Delta c c / A$ \\
\hline Fbre3R & ACAACAAAACTAGCTCTCTCGGC & 3' Flank $\Delta c c l A$ \\
\hline FbreCOMF-Notl & TTGCTCCAGCGGCCGCATCCTCAAACCTCGCCAAGTATG & cclA complementation \\
\hline FbreCOMR-Spel & GGAAATACTAGTGAAGCTGAAAGTGACGTCTAGAA & cclA complementation \\
\hline JP1 & TATAGGGACGAATTCACAGACAATG & 5' Flank $\Delta h d m A$ \\
\hline JP2 & CGCTGAATTCGGGTTTGATGGACATTGGAC & 5' Flank $\Delta h d m A$ \\
\hline JP3 & CCGGICTAGAGGTAATGCTTAGACTCCCGTA & 3' Flank $\Delta h d m A$ \\
\hline JP4 & GTCATGCGGCCGCCACTGCCCTCGTTAAGG & 3' Flank $\Delta h d m A$ \\
\hline JP IsdA comp For & GCTTGGATCCGACGTAGCAGGTGAAC & hdmA complementation \\
\hline JP IsdA comp Rev & CATTGGATCCTCCTCGCCTTTCTCC & hdmA complementation \\
\hline JP Afumi argB For & GAACGCGGTCTGCATCCAAG & $A f$-argB cloning \\
\hline JP Afumi argB Rev & GAAGGAGAGACCCATACATCC & $A f$-argB cloning \\
\hline FumlaeAF & ATGTTTCTCAACGGGCAGGGC & laeA probe \\
\hline FumlaeAR & ATTGGCGAGAGGTTTTCGAGCC & laeA probe \\
\hline FbrelF & CTTTCCACACATCAAGTACCGGC & cclA probe \\
\hline FbrelR & ATTGAAGCGTTCGCCAATGCC & cclA probe \\
\hline JP LSD1 & TCTCACGCAACTACATACGTCAAC & hdmA probe \\
\hline JP LSD2 & TTTTTCCTCTTCGCTGGCTTGCC & hdmA probe \\
\hline GZINTF & AAGGGCCGGTAGTCTACCTCTTC & gliz probe \\
\hline GZINTF & CGATCTGGTAGCTGCCCAGCTGGAAG & gliz probe \\
\hline
\end{tabular}




\section{Figure 1}

Construction of deletion and subsequent complementation strains of cclA and $h d m A$ in $A$. fumigatus.
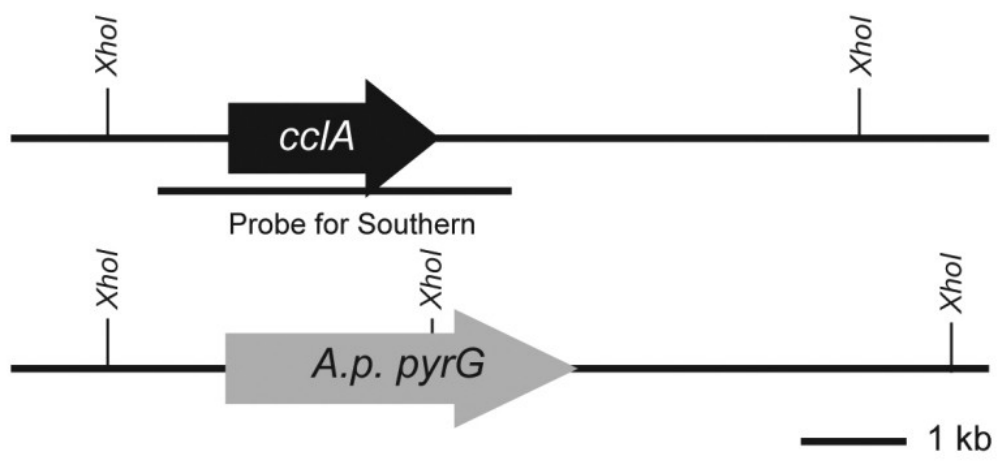

$1 \mathrm{~kb}$
B

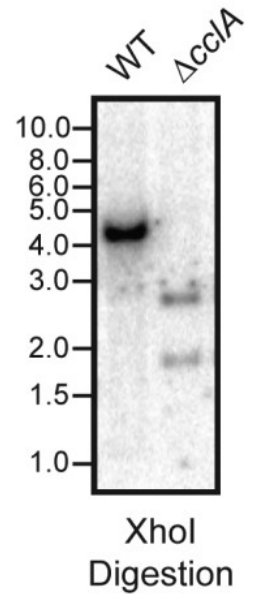

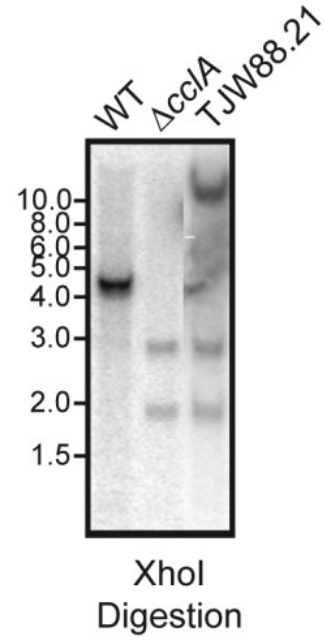

c
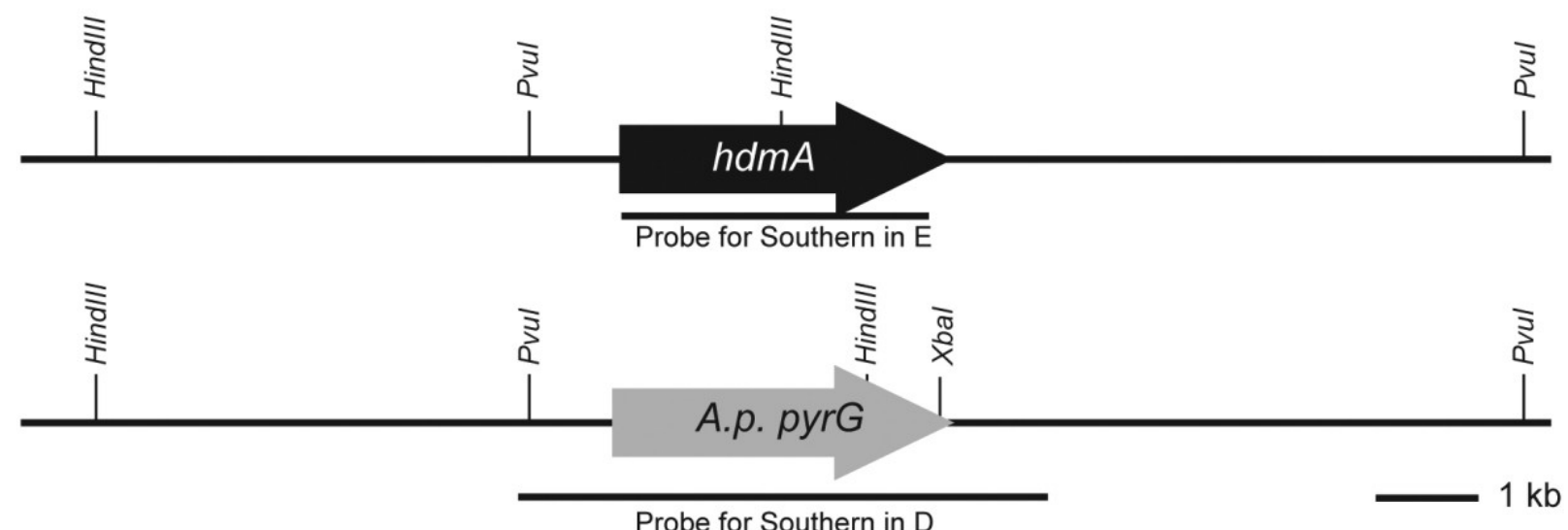

D

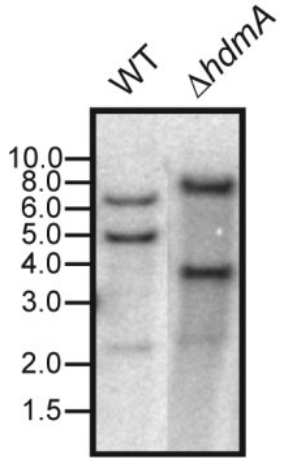

HindIII

Digestion

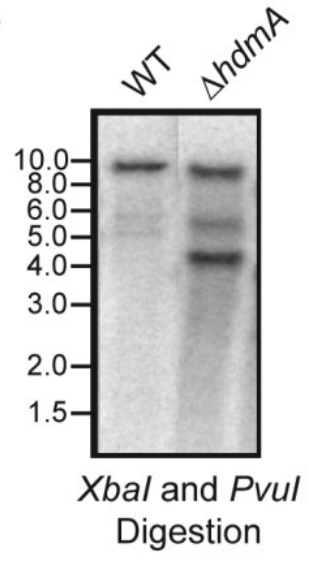

E

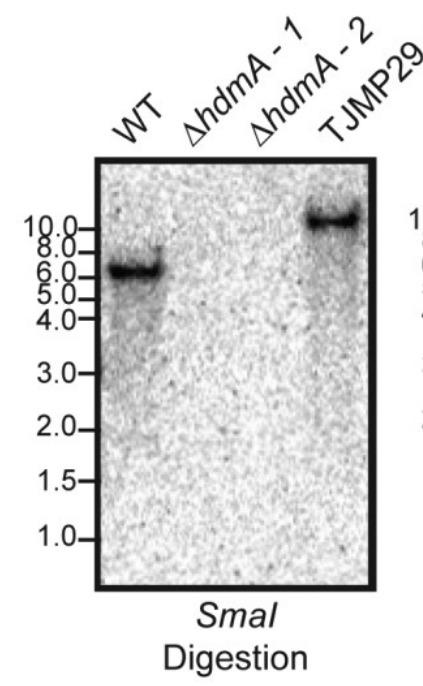

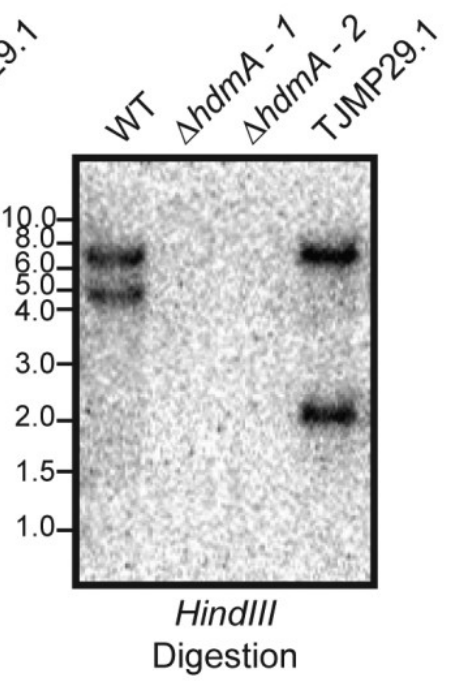




\section{Figure 2}

Deletion of cclA results in a reduction in di-methylation and loss of tri-methylation of lysine 4 of histone 3 (H3K4).

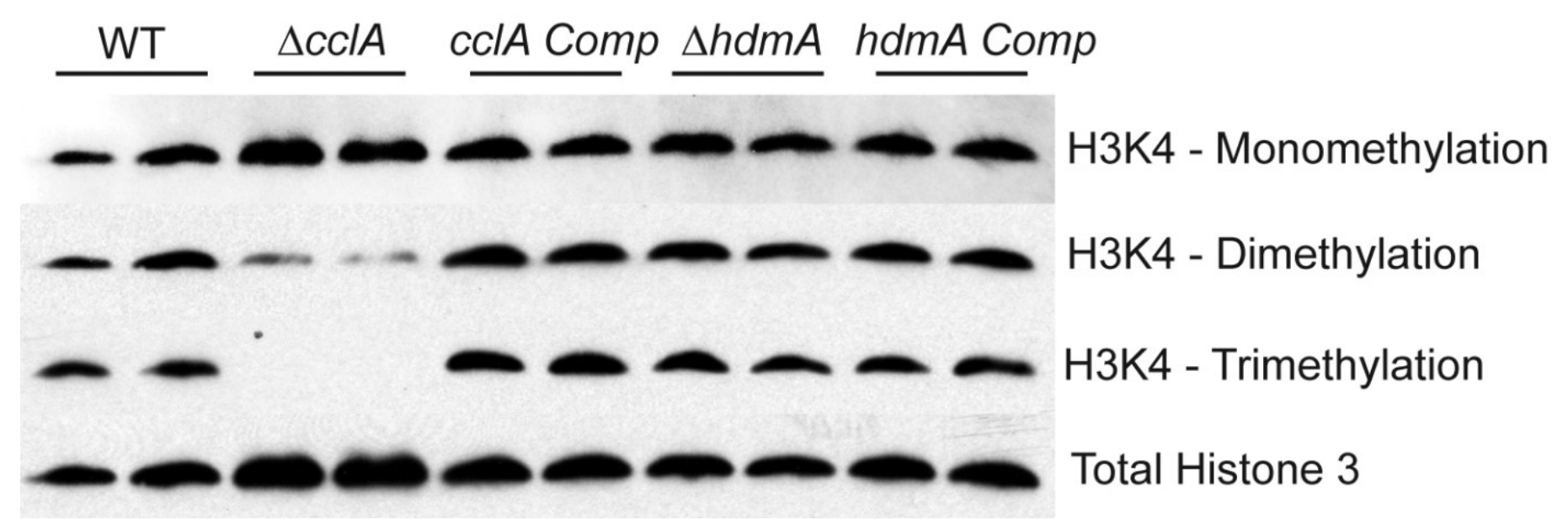




\section{Figure 3}

Null mutants of cclA result in reduced growth and increased sensitivity to 6-azauracil.
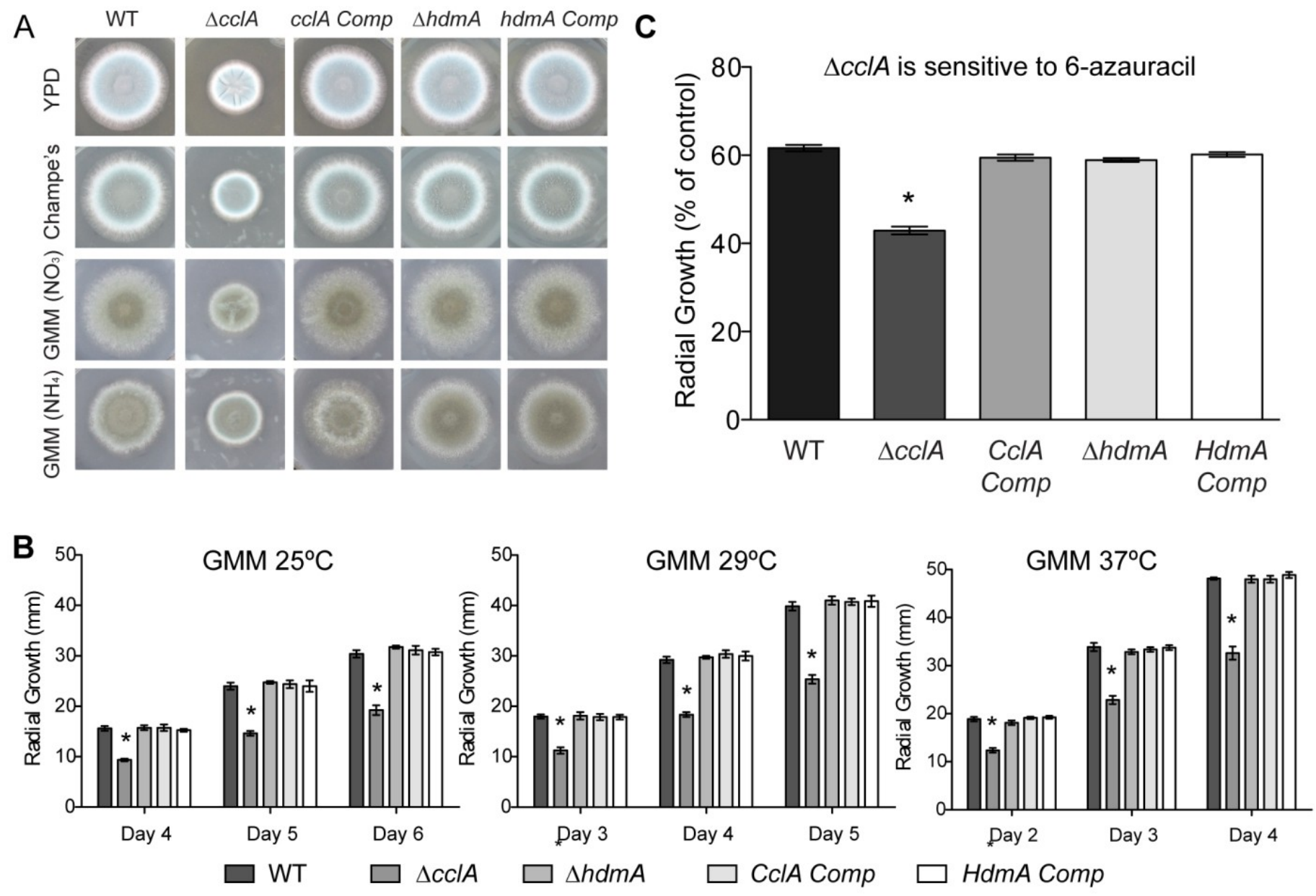


\section{Figure 4}

CclA, but not HdmA, controls production of several secondary metabolites including gliotoxin.
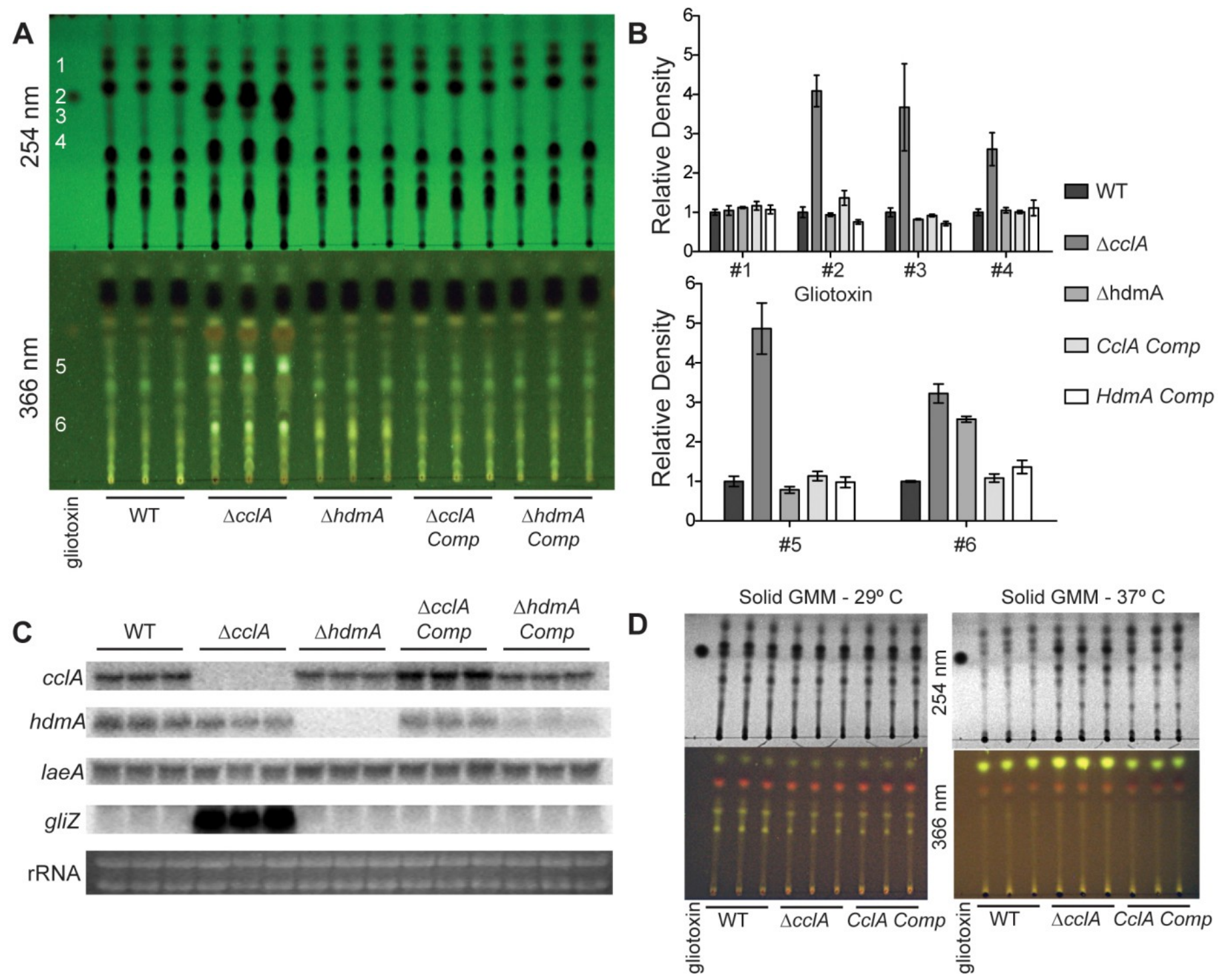


\section{Figure 5}

Pathogenicity of $\triangle c c l A$ and $\Delta h d m A$ strains are wild type in murine model of IA and $\Delta c c l A$ strains are wild type in Toll-deficient Drosophila model. 
A

Neutropenic Mouse Model

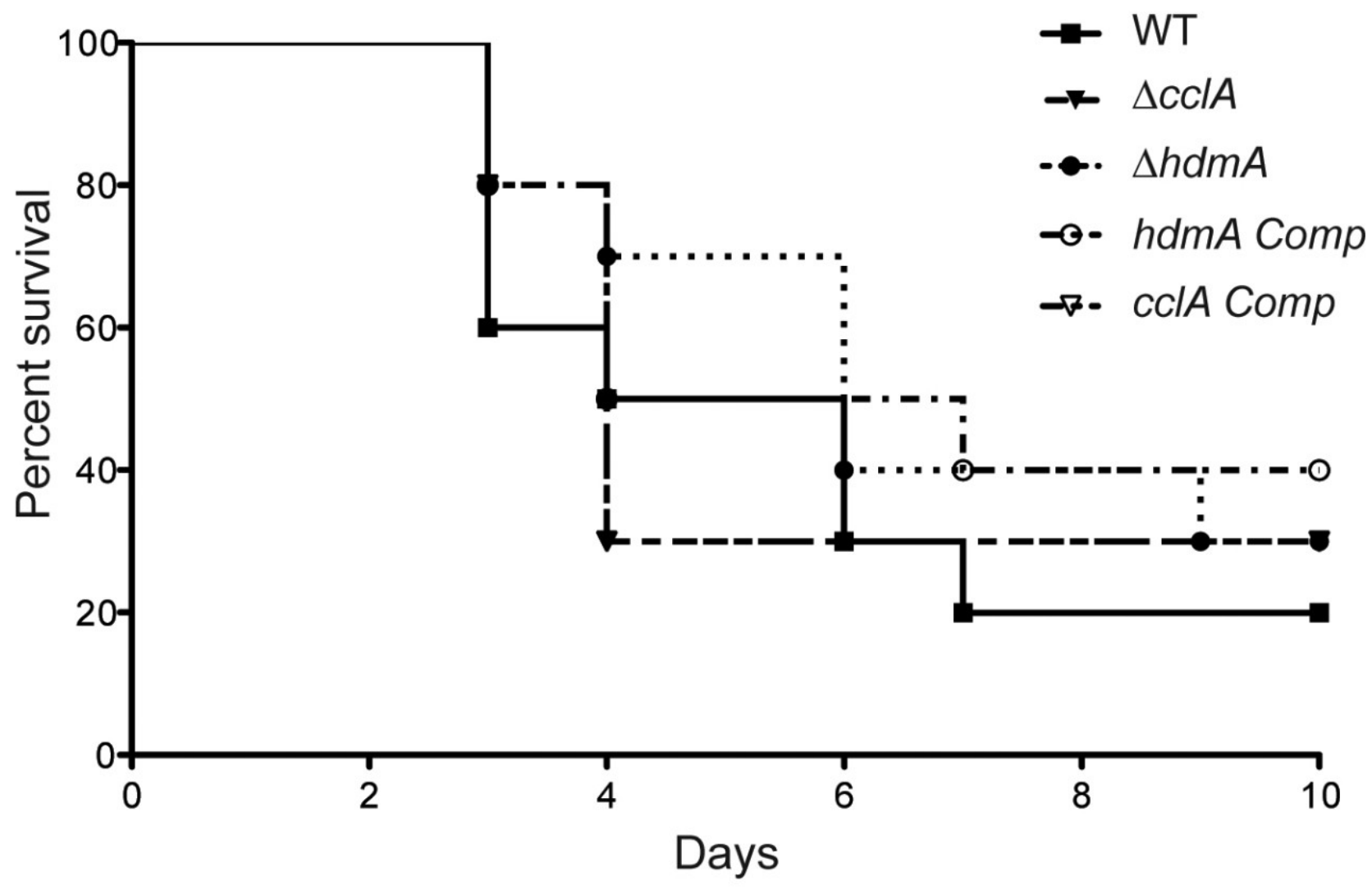

B

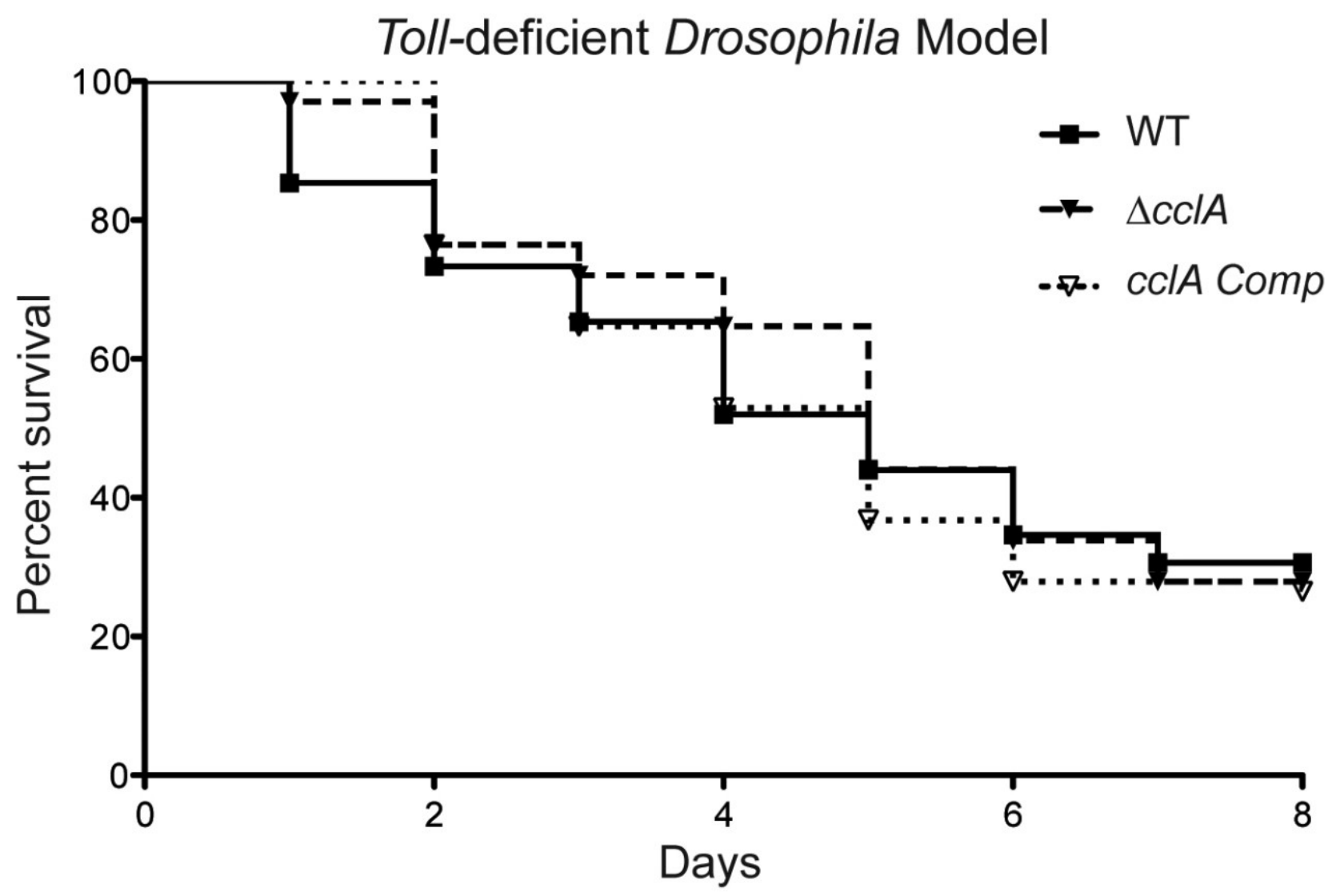

